\title{
Influence of Te concentration on the infrared cathodoluminescence of GaAs:Te wafers
}

\author{
B. Mendez and J. Piqueras \\ Departamento de Fisica de Materiales, Facultad de Fisicas, Universidad Complutense, \\ 28040 Madrid, Spain
}

(Received 25 June 1990; accepted for publication 28 November 1990)

Cathodoluminescence (CL) scanning electron microscopy has been used to investigate the nature and distribution of defects involved in the infrared emission of GaAs:Te wafers. Spectral and CL-contrast changes as a function of doping level have been found. Profiles of infrared $C L$ intensity across the wafers show an inverted $U$ shape.

\section{INTRODUCTION}

The distribution of defects, impurities, and of several physical parameters is known to be inhomogeneous in III-V wafers, showing often W-, M-, or U- shaped profiles across a wafer diameter. One of the techniques that provide information about space distribution and nature of defects, or other recombination centers in III-V wafers is the cathodoluminescence (CL) in the scanning electron microscope (SEM). In the case of GaAs, previous CL-SEM work refers mainly to near- band-edge luminescence and sometimes in semi-insulating material to infrared region. ${ }^{1,2}$ However, the uniformity of CL emission and of defect concentration across $n$-doped GaAs wafers has been only seldom reported. In a previous work ${ }^{3}$ the spatial distribution of defects in GaAs:Te wafers was investigated by measuring near-band-edge $C L$. On the other hand the nature of defects responsible of some luminescence bands in the near-infrared region in $n$-type GaAs has been investigated ${ }^{4-7}$ but no imaging techniques were used. In the present work near-infrared CL-SEM is used to study the nature and space distribution of defects in GaAs:Te wafers with different doping levels.

\section{EXPERIMENTAL METHOD}

The samples used in this study were Te- doped (100) oriented $\mathrm{GaAs}$ wafers of $50-\mathrm{mm}$ diameter. Three wafers, hereinafter denoted as $A, B$, and $C$, with free-carrier concentrations $n$ of $2.2 \times 10^{17} \mathrm{~cm}^{-3}, 4.5 \times 10^{17} \mathrm{~cm}^{-3}$, and 1.5 $\times 10^{18} \mathrm{~cm}^{-3}$, respectively, were investigated. The measurements were done on $5-\mathrm{mm}$-wide strips, containing the center of the wafer, which were cut with a diamond saw along the wafer diameter. For the observations in the SEM each strip was cut in ten parts each about $5 \times 5 \mathrm{~mm}{ }^{2}$ When comparison of the $\mathrm{CL}$ emission of the ten parts was made, in order to obtain a CL intensity profile across the wafer diameter, all the samples were placed in a single specimen holder and measurements were made under the same experimental conditions. The samples were observed in a $\mathrm{Hi}-$ tachi S-2500 or in a Cambridge S4-10 scanning electron microscope at $30 \mathrm{keV}$ at temperatures between 160 and 273 $\mathrm{K}$ in the emissive and CL modes. The luminescence was measured either with a photomultiplier covering the range $380-850 \mathrm{~nm}$ or with a North Coast EO-817 germanium detector in the range $0.8-1.8 \mu \mathrm{m}$. When obtaining pan- chromatic images a lens was used to concentrate the light on the detection unit attached to a window of the microscope. To record spectra a light guide feeding the light to an Oriel 78215 computer-controlled monochromator was used. The spectra were corrected to include the system spectral response. Infrared monochromatic imagcs related to the main emission band could be obtained. Furthermore, total infrared images were recorded by using a 1000 $\mathrm{nm}$ cuton filter that suppresses contribution from the nearband-edge emission.

\section{RESULTS}

CL spectra have been found to depend on the doping and on the temperature. Figure 1 summarizes the results for the different cases. The fundamental band has been included as a reference but in the following we describe only the emission at lower energies (IR band). At room temperature the $A$ sample (lower Te concentration) shows a band at about $1300 \mathrm{~nm}$ while the spectra of samples $B$ and $C$ are almost featureless. The spectra of Fig. 1(a) have been recorded under the normal observation conditions of the SEM, with the electron beam focused on the sample. With a defocused beam the spectrum of sample $C$ does not show new features while in sample $A$ the $1300 \mathrm{~nm}$ band is maximized and in sample $B$ a band at about $1260 \mathrm{~nm}$ emerges (Fig. 2). At low temperatures [Fig. 1(b)] the three samples show infrared bands even with a focused electron beam. In sample $A$ the band is centered at about $1260 \mathrm{~nm}$ and a shift to higher energies is observed by increasing doping.

The IR-CL images have been also found to depend on the Te concentration. In sample $A$ infrared and visible $C L$ images have the same appearance showing dark striations interpenetrated by an ill-defined cellular structure with dark walls and bright cell interiors. In some regions dark spots of about 3-5 $\mu \mathrm{m}$ size are also present (Fig. 3). Images of samples $B$ and $C$ show common features. The image corresponding to the near-band-edge emission shows, in both samples, the well known dot and halo contrast related to the presence of dislocations and defects surrounding them. On the contrary in the infrared images bright spots are observable. Figure 4 shows near-band-edge luminescence and infrared images of the same area of a $C$ sample. Inspection of both micrographs reveals that the 

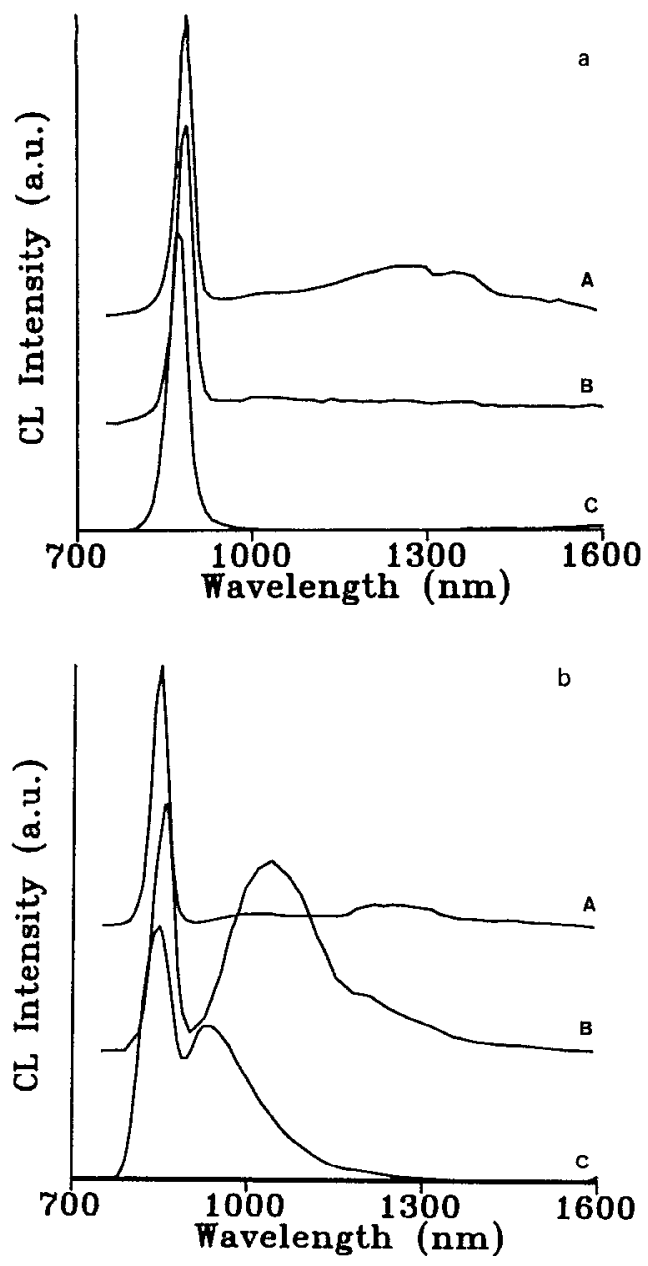

FIG. 1. CL spectra from Te-doped GaAs wafers. (a) room temperature, and (b) $160 \mathrm{~K}$.

blurred bright spots in the infrared emission correspond to dark spots or spot groups in the fundamental emission distribution. Some of such correspondences have been marked in the micrographs. A feature, only observed in $C$ samples, is the presence, in the near-band-edge CL image,

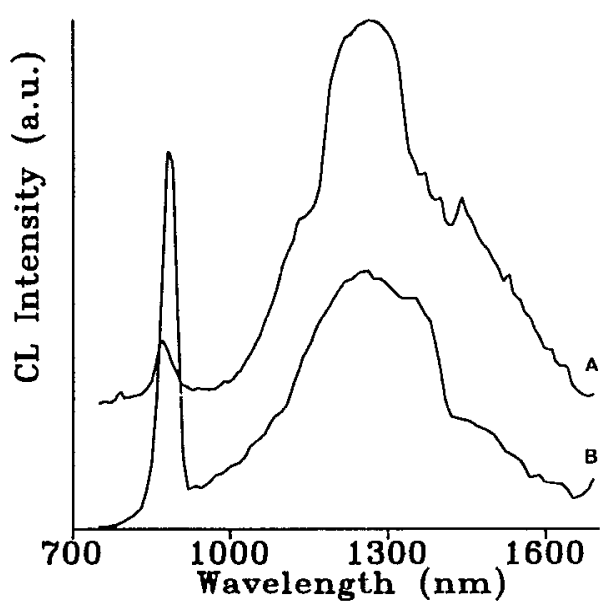

FIG. 2. CL spectra recorded with defocused electron beam
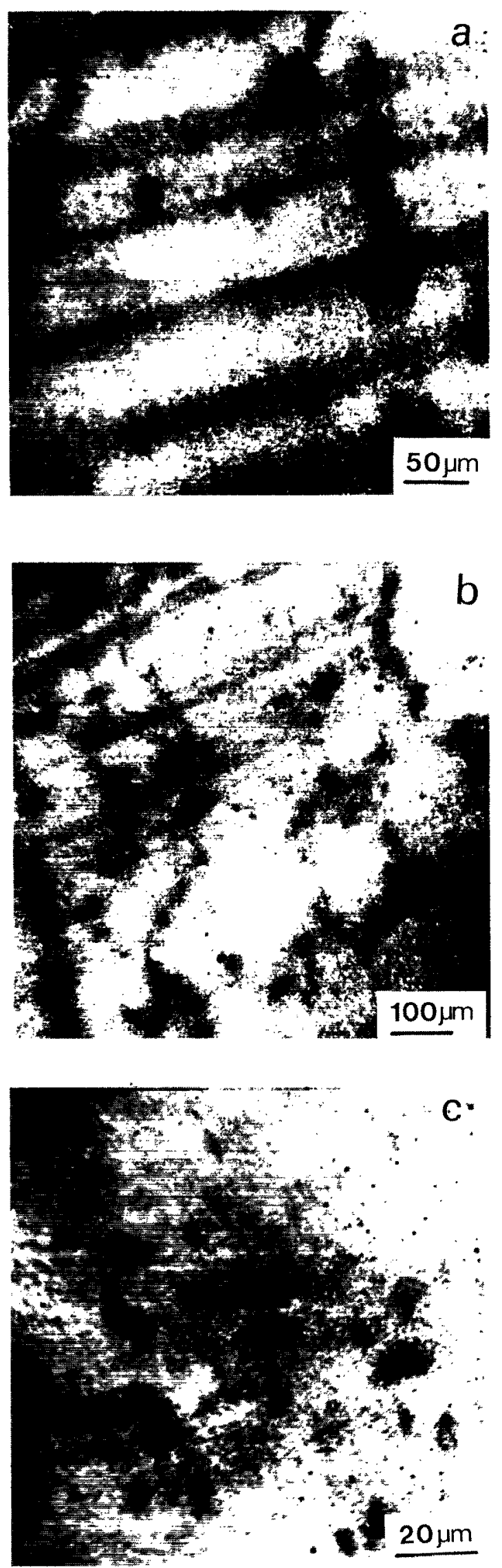

FIG. 3. CL image of sample $A$ showing (a) striations, (b) cellular structure, and (c) dark spots in dark walls. 

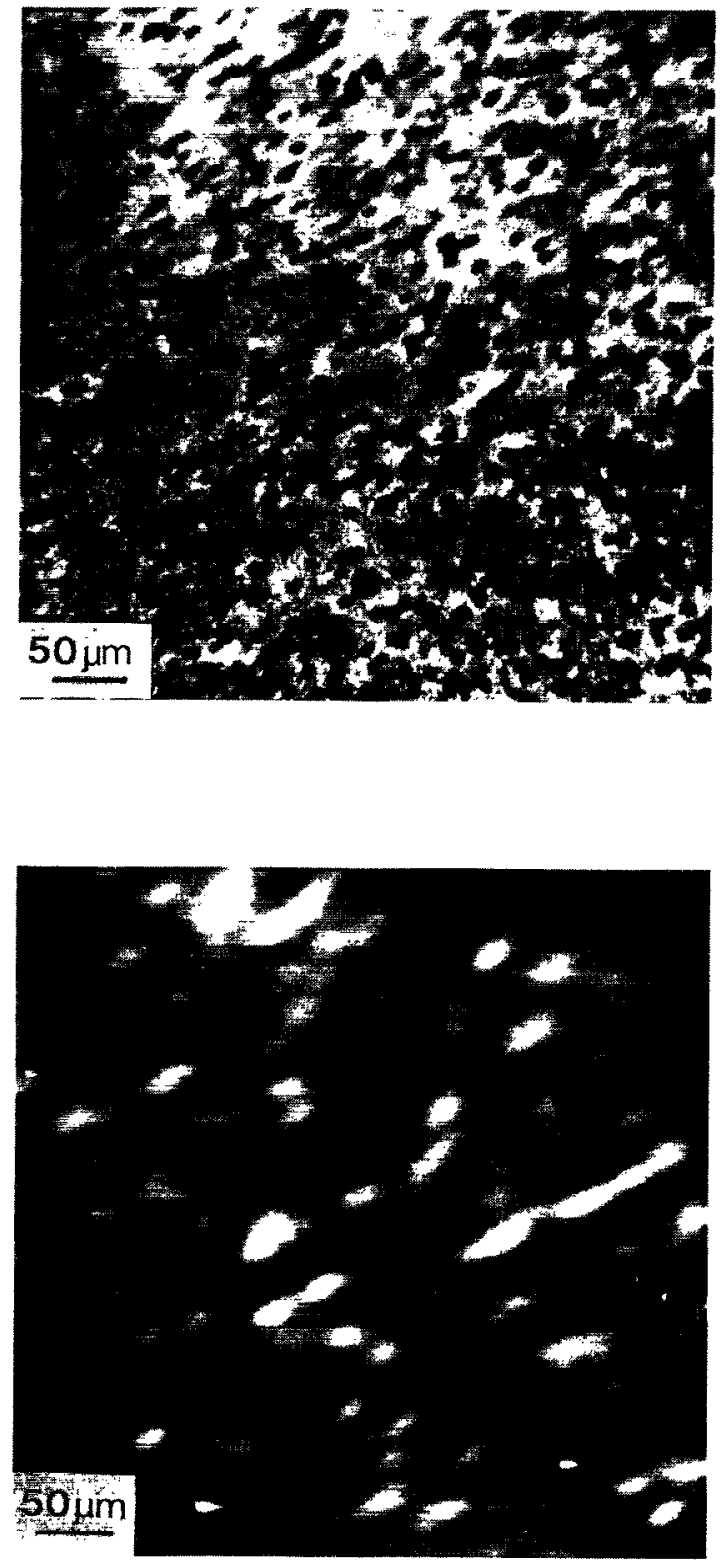

FIG. 4. CL image of sample $C$ at low temperature (a) near-band-edge luminescence image, and (b) IR band luminescence image.

of a high number of a different kind of dark spots. These are smaller than those surrounded by halo as the fine background of nondislocation-related dark spots described by Ref. 7 in moderate to high Te-doped crystals. Dark spots related to precipitates have been also described in Ref. 8 in Te-doped GaAs. They can be observed in Fig. 5 in the dot and halo-free areas but are not resolved in the infrared images.

Figure 6 shows CL intensity profiles across a wafer diameter measured in both spectral regions. The band-edge CL shows in all three samples the U-shaped profile that has been previously described ${ }^{1}$ while the infrared-CL profiles have an inverted $U$ shape. The IR profiles could only be measured in $A$ and $B$ samples because the infrared emission was too low in sample $C$.

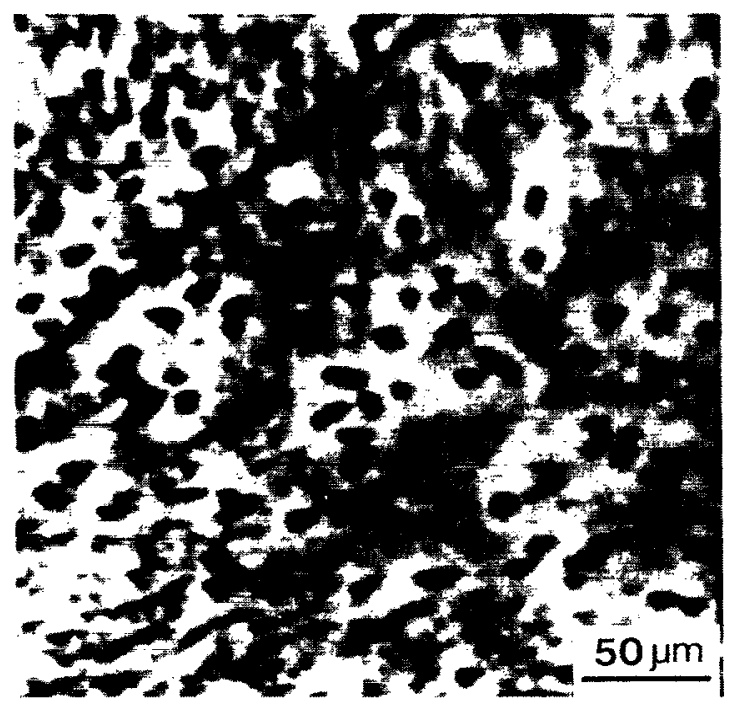

FIG. 5. Total CL image of sample $C$.

\section{DISCUSSION}

In previous works ${ }^{4-7}$ the existence of a luminescence band in the region $1.2-1.3 \mathrm{eV}(1030-930 \mathrm{~nm})$ in GaAs:Te crystals has been described. In the mentioned works the infrared luminescence band has been attributed to impurity-point-defect complexes as $\mathrm{Te}_{\mathrm{As}_{\mathrm{s}}}-\mathrm{V}_{\mathrm{Ga}}$. A theoretical model of such complexes has been treated by Hurle ${ }^{9}$ while other complexes involving both kinds of vacancies, $\mathrm{Te}_{\mathrm{As}}-\mathrm{V}_{\mathrm{Ga}}-\mathrm{V}_{\mathrm{As}}$, have been proposed by Hughes and Narayanan ${ }^{10}$ based on transmission electron microscopy results. On the other hand Markov et al. ${ }^{11}$ in a study of microdefects near dislocations in GaAs:Te take into account the presence of $\mathrm{Te}_{\mathrm{As}}-\mathrm{V}_{\mathrm{Ga}}$ complexes to explain the formation of different types of microdefects in strongly Te-doped crystals. These kinds of microdefects act as electron-hole recombination centers competing with the near-band-edge transition. By defocusing the electron beam, deep levels are unsaturated, so recombinations involving them are more

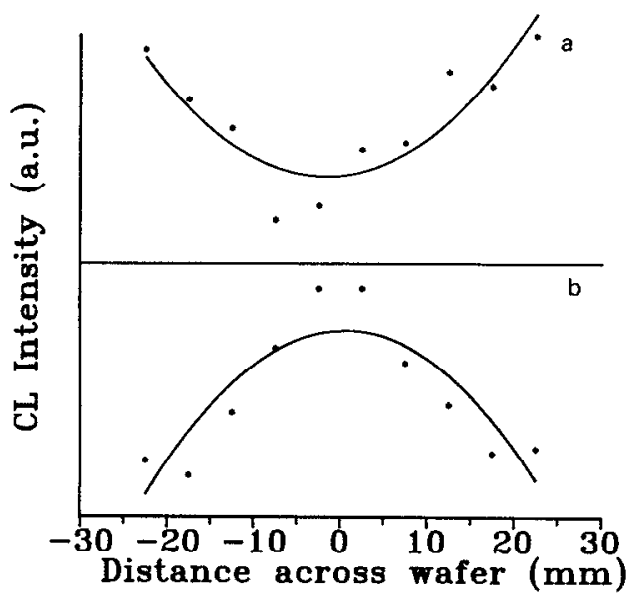

FIG. 6. (a) Profile of the near-band-edge luminescence and (b) profile of the IR band luminescence across the diameter of wafer $A$. 
efficient than near-band-edge transition, as is shown in Fig. 2. The exact position of the band peak has been found in Ref. 7 to depend on the doping level $(1.2 \mathrm{eV}$ for $n<2 \mathrm{x}$ $10^{18} \mathrm{~cm}^{-3}$ and $1.3 \mathrm{eV}$ for $n>2 \times 10^{18} \mathrm{~cm}^{-3}$ ) whilc in the other mentioned works no shift of the IR band with doping is reported. In the present work a clear shift of the band peak to higher energies by increasing the $n$ value has been observed. The peak energy moves from 1 to $1.33 \mathrm{eV}$ in the three samples investigated but the halfwidth has been found to be the same for samples $A$ and $B$ and only slightly higher in sample $C$. The halfwidth in $A$ and $B(181 \mathrm{meV})$ has been reported in Ref. 4 for the $1.2 \mathrm{eV}$ band. A mechanism for the energy shift of the IR band has not yet been given. However, the formation of different kinds of defects, impurity-vacancy complexes, as a function of doping level, as discussed in Ref. 11 can contribute to the observed effect. This possibility is supported by CL observations. The $\mathrm{CL}$ images indicate that the spatial distribution of the IR emission in sample $A$ has features not observed in samples $B$ and $C$. In $A$ the defects responsible for the $1 \mathrm{eV}(1240$ $\mathrm{nm}$ ) emission have the same distribution that defects producing contrast in the near-band-edge $\mathrm{CL}$ image. As described above, both images have the same appearance. On the contrary in $B$ and $C$, the defects responsible for the IR band give rise to a $C L$ image almost opposite to that of the near-band-edge emission as Fig. 4 shows. On the other hand, a common feature to all samples is that defects involved in the IR band act as competitors of the near-bandedge luminescence. This is apparent in the opposite profiles of the intensities of both bands across the wafer diameter (Fig. 6).

Other features observed in the different crystals investigated here are determined by the growth conditions and by the $\mathrm{Te}$ content. The latter certainly produces the formation of the small dark defects in the band edge CL image of sample $C$. A fine background of dark defects has been described ${ }^{7}$ in samples with high Te concentration. Our observations show that the small defects are present even if the dislocation density is higher than $10^{3} \mathrm{~cm}^{-2}$, contrary to that observed in Ref. 7, and on the other side the defects have not been found to contribute to the IR image. Finally in sample $A$, striations, cellular structure, and spots produce a dark contrast in both images. It is known that striations and other features are related to doping inhomogeneity that probably appeared in this sample due to the low doping level and possibly specific growth conditions.

\section{CONCLUSIONS}

The peak energy of an infrared band in the range $1-1.33 \mathrm{eV}$ has been found to depend on the Te concentration $\left(2 \times 10^{17}\right.$ to $\left.1.5 \times 10^{18} \mathrm{~cm}^{-3}\right)$ in GaAs:Te wafers. The emission is thought to be related to impurity-vacancy complexes. Infrared CL images reveal an almost inverse contrast to that observed in band edge CL images in highly doped samples. Profiles of the infrared luminescence across the wafer diameter have been found to have an inverse $U$ shape.

\section{ACKNOWLEDGMENTS}

This work was supported by the Comision Interministerial de Ciencia y Tecnologia (Project PB86-0151) and by DGICYT-DAAD. The authors thank WackerChemitronic (Dr. K. Löhnert) for providing the samples.

${ }^{1}$ M. Dussac, M. Dupuy, and E. Molva, Defect Recognization and Image Processing in III-V Compounds, edited by J.P. Fillard (Elsevier, Amsterdam, 1985), p. 209.

${ }^{2}$ C. A. Warwick and G. T. Brown, Appl. Phys. Lett. 46, 574 (1985).

${ }^{3}$ B. Méndez, J. Piqueras, F. Domínguez-Adame, and N. de Diego, J. Appl. Phys. 64, 4466 (1988).

${ }^{4}$ E. W. Williams, Phys. Rev. 168, 922 (1968).

${ }^{5}$ C. J. Hwang, J. Appl. Phys. 40, 4584 (1969).

${ }^{6}$ C. J. Hwang, J. Appl. Phys. 40, 4591 (1969).

${ }^{7}$ A. V. Govorkov and L. I. Kolesnik, Sov. Phys. Semicond. 12, 259 (1978).

${ }^{8}$ Y. M. Chu, D.B. Darby, and G. R. Booker, Inst. Phys. Conf. Ser. No. 60, 331(1981).

${ }^{9}$ D. T. J. Hurle, Inst. Phys. Conf. Ser. 33a, 113 (1977).

${ }^{10}$ B. Hughes and G. H. Narayanan, Phys. Status Solidi A 46, 627 (1978).

"A. V. Markov, M. G. Milvidskii, and S.S. Shifrin, Sov. Phys. Crystallogr. 29, 205 (1984) 
Journal of Applied Physics is copyrighted by the American Institute of Physics (AIP). Redistribution of journal material is subject to the AIP online journal license and/or AIP copyright. For more information, see http://ojps.aip.org/japo/japcr/jsp

Copyright of Journal of Applied Physics is the property of American Institute of Physics and its content may not be copied or emailed to multiple sites or posted to a listserv without the copyright holder's express written permission. However, users may print, download, or email articles for individual use. 